\title{
Primary brachytherapy as a radical treatment for endometrial carcinoma
}

\author{
Elzbieta van der Steen-Banasik, MD \\ Department of Radiation Oncology. Arnhem Radiotherapy Institution (ARTI), Arnhem, The Netherlands
}

\begin{abstract}
Surgery is the primary treatment for endometrial cancer, with radiation therapy having an established place in the adjuvant treatment setting. However, there is a population of patients with endometrial cancer who may not be suitable for surgery and primary radiation therapy as a treatment option. This article describes the place of radiotherapy in the management of endometrial cancer and describes the author's own experiences with primary brachytherapy in those patients who are unsuitable for surgery.

Key words: brachytherapy, endometrial cancer, HDR.

\section{Purpose}

Endometrial cancer is the most common malignancy of the female reproductive tract in developed countries. The highest incidence rates are in Western Europe and North America with world age standardized incidence per 100,000 being 11.2 and 16.4, respectively in 2008, compared to 1.9 in Western and Middle Africa and a world incidence of 8.2 [1]. The incidence rises with increasing age and peaks in the sixth and seventh decade. Obesity is a well-recognized risk factor, increasing the risk of developing endometrial cancer three-fold. This specific relationship has been explained by alterations in hormone metabolism in obese women [2]. Endometrial cancer incidence has slowly increased over recent years. In the Netherlands, the incidence has increased from nearly 1600 new cases per year at the beginning of the $21^{\text {st }}$ century to between 1800 and 1950 new cases per year in the last 5 years. The incidence in elderly patients ( $>85$ years) is approximately 150 new cases per year, and in patients aged above 90 years it is 30 new cases per year [3].

\section{General principles for the treatment of endometrial cancer}

The treatment of choice is surgery: total abdominal or laparoscopic hysterectomy with bilateral salpingo-oophorectomy (TAH/TLH/BSO). The necessity of systematic lymphadenectomy is subject to discussion. Two large randomized studies did not demonstrate survival nor recurrence rate benefit of lymph node dissection (LND), however, the investigated patient population included predominantly intermediate risk tumors $[4,5]$. That is why in general LND is being performed in the case of high risk tumors: FIGO stage 1B grade 3 and higher. The Clinical, TNM, FIGO 2009, and surgical pathological staging of endometrial cancer are shown in Table 1.

The role of adjuvant radiation therapy has been established as a result of a number of essential randomized trials [6-9]. Postoperative external beam pelvic radiotherapy (EBRT) remains indicated for high risk and advanced disease, whilst vaginal brachytherapy (VBT) achieves excellent vaginal control and survival, and recurrence rates are similar to EBRT. Therefore, VBT is the adjuvant treatment of choice for patients with high-intermediate risk disease [10-12]. Subsequently chemotherapy was added, based upon the results of two recent randomized studies, in which adding chemotherapy in high risk patients showed improvement of 5 -year progression free survival from $69 \%$ to $78 \%$ [13]. Current ongoing studies are further investigating this subject (PORTEC 3, GOG 249, GOG 258). The outcome of various randomized studies resulted in evidence based guidelines is illustrated in Table 2 . The reported 5-year survival rates are $80 \%$ for the whole population, $85-90 \%$ for stage I, $75-85 \%$ for stage II, $50-65 \%$ for stage III, and $20-25 \%$ for stage IV [14]. Approximately $55 \%$ of patients present with early stage, low risk tumors with a relapse free survival rate of $95 \%$ after primary surgical treatment and for this population adjuvant treatment is not indicated [15].

\section{Primary brachytherapy in endometrial cancer}

There is an increasing population of aging or morbidly obese patients who cannot undergo any form of anesthesia, and as a consequence, in whom surgery is considered
Address for correspondence: Elzbieta van der Steen-Banasik, MD, Department of Radiation Oncology, Arnhem Radiotherapy Institution (ARTI), Wagnerlaan 47, 6815 AD Arnhem, The Netherlands, phone: 0031263712412, $₫$ e-mail: E.vanderSteen-Banasik@arnhemrti.nl
Received: 04.01.2014

Accepted: 28.03.2014

Published: 28.03.2014 
Table 1. Staging of endometrial cancer

\begin{tabular}{lccl} 
Clinical & TNM & FIGO 2009 & FIGO Corpus Uteri 2009 \\
\hline I & T1 & I & Tumor confined to corpus uteri \\
& T1a & IA & No or less than $50 \%$ myometrial invasion \\
& T1b & IB & More than $50 \%$ myometrial invasion \\
\hline II & T2 & II & Tumor invades cervical stroma, but does not extend beyond the uterus \\
\hline III & T3 and/or N1 & III & Local and/or regional spread of the tumor \\
& T3a & IIIA & Tumor invades the serosa and/or adnexae \\
T3b & IIIB & Vaginal and/or parametrial involvement \\
& T1-3 N1 & IIIC & Metastases to pelvic and/or para-aortic lymph nodes \\
& IIIC & Positive pelvic nodes \\
& IIIC & Positive para-aortic nodes with or without positive pelvic nodes \\
\hline IV & IV & Tumor invades bladder and/or bowel mucosa, and/or distant metastases \\
& IVa & Invasion of bladder/bowel mucosa \\
& T4 and/or M1 & IVb & Distant metastases, including intra-abdominal metastases and/or inguinal \\
& lymph nodes
\end{tabular}

Table 2. Treatment of endometroid type endometrial cancer according to Dutch guidelines 2011 [11]

\begin{tabular}{|c|c|c|c|c|}
\hline FIGO 2009 & Grade & Age (years) & Surgery & Adjuvant therapy \\
\hline \multirow[t]{3}{*}{ IA } & 1,2 & All & $\mathrm{TAH} / \mathrm{TLH}+\mathrm{BSO}$ & No \\
\hline & 3 & $<60$ & $\mathrm{TAH} / \mathrm{TLH}+\mathrm{BSO}$ & No (unless LVSI1: vaginal brachytherapy) \\
\hline & 3 & $\geq 60$ & $\mathrm{TAH} / \mathrm{TLH}+\mathrm{BSO}$ & $\begin{array}{l}\text { No (unless myometrium invasion: vaginal } \\
\text { brachytherapy) }\end{array}$ \\
\hline \multirow[t]{3}{*}{ IB } & 1,2 & $<60$ & $\mathrm{TAH} / \mathrm{TLH}+\mathrm{BSO}$ & No \\
\hline & 1,2 & $\geq 60$ & $\mathrm{TAH} / \mathrm{TLH}+\mathrm{BSO}$ & Vaginal brachytherapy \\
\hline & 3 & & $\mathrm{TAH}+\mathrm{BSO}+\mathrm{LND}$ & Vaginal brachytherapy \pm chemotherapy \\
\hline II & $1,2,3$ & All & $\begin{array}{l}\text { Radical hysterectomy + LND } \\
\mathrm{TAH}+\mathrm{BSO}\end{array}$ & $\begin{array}{l} \pm \text { Chemotherapy } \\
\text { Combination of external beam radiotherapy } \\
\text { and vaginal brachytherapy } \pm \text { chemotherapy }\end{array}$ \\
\hline $\begin{array}{l}\text { IIIA } \\
\text { IIIB } B_{\text {parametial }}\end{array}$ & $1,2,3$ & All & TAH + BSO, macroscopically radical & External beam radiotherapy \pm chemotherapy \\
\hline IIIB $\mathrm{B}_{\text {vagina }}$ & $1,2,3$ & Vagina & $\begin{array}{l}\text { TAH + BSO, macroscopically } \\
\text { nonradical } \\
\text { Irresectable }\end{array}$ & $\begin{array}{l}\text { Combination of external beam radiotherapy } \\
\text { and vaginal brachytherapy } \\
\text { Combination of external beam radiotherapy } \\
\text { and brachytherapy, possibly followed by hys- } \\
\text { terectomy }\end{array}$ \\
\hline$\left\|I C_{1} /\right\| C_{2}$ & $1,2,3$ & All & $\mathrm{TAH}+\mathrm{BSO} \pm \mathrm{LND}$ & External beam radiotherapy \pm chemotherapy \\
\hline IVA/IVB & $1,2,3$ & & & Palliation \\
\hline
\end{tabular}

LVSI - lymph vessel space invasion

too high a risk. For these patients, hormonal treatment (eg. Mirena) can offer a solution, however only temporarily. When the symptoms of the cancer recur, the patient is even older and more fragile or vulnerable. For this population radical radiotherapy provides a solution with a high cure rate, and is also indicated in patients with an irresectable tumor. In 1947, the role of primary radiotherapy in endometrial cancer had already been described by Heyman [16]. Several authors have subsequently described their experience using radium and low dose rate (LDR) [17-23]. In recent years, high dose rate (HDR) treated patient populations were also reported [24-30]. Nowadays different types of HDR applicators are available: modified Heyman packing, one channel applicators, and rotte $\mathrm{Y}$ applicators.

The effectiveness of HDR based primary radiotherapy for endometrial carcinoma was demonstrated in the results of several single institution reports that documented a 5-year disease specific survival (DSS) of up to $80 \%$ [2430]. Coon et al. described in 2008 a 5 -year DSS of $87 \%$ for a population of 47 patients with stage 1 disease and above, using a combination of external beam radiation and brachytherapy with a Rotte applicator [24]. Another significant population $(N=280)$ was described by the Vienna group, with slightly inferior results with a 5-year DSS of $76 \%$. However, in this later study an inferior applicator, a vaginal cylinder with one single intrauterine device was used [26]. After the introduction of the Heyman packing, improved results were seen: 16 patients in one study with a 4-year DSS of $100 \%$ [27]. Modified Heyman packing (Norman Simon applicators) consists of long thin flexible tubes available with capsules of different sizes at their top for packing in the uterine fundus and horns. This allows adaptation to the individual anatomy of the uterine cavity. The number of tubes applied varies 
between 5 and 18, depending on the individual anatomy. This technique results in adequate, probably even optimal dose distribution in the uterine corpus [31]. This Heyman approach demonstrates excellent results, however the packing can only be inserted under anesthesia and is therefore not suitable for the population described above, where anesthesia is contraindicated due to comorbidities. In Table 3 some chosen results of HDR brachytherapy as primary treatment of endometrial cancer are presented. The toxicity differs, depending upon whether external beam is a part of the treatment or brachytherapy alone is applied. In the image guided brachytherapy alone series, the acute and late toxicity rate is very low, around $10 \%$ and predominantly grade 1 and 2 [23-30].

\section{Primary brachytherapy treatment description based on own experiences}

The Arnhem Radiotherapy Institute (ARTI) is specialized in brachytherapy, including brachytherapy for inoperable endometrial cancer. In our institution, we chose the Y-shaped Rotte applicator for this treatment, which we have used since 1995 and have successfully treated a total of 20 patients. This applicator comes in 4 sizes and can be completed with an additional vaginal cylinder, if the vagina is considerd to be part of the clinical target volume (CTV). The optimal coverage of the uterus is obtained by choosing the most appropriate applicator size. The correct applicator size is confirmed during the preplannig stage, based on imaging, preferably made by MRI scan or diagnostic CT-scan. The Y Rotte applicator is easy to insert without anesthesia. If necessary, a low dose tranquilizer provides sufficient premedication. Unfortunately, the applicator is not MRI compatible and for definitive planning purposes a CT-scan is the only suitable imaging.
At ARTI we have recently observed an increase in the number of patients who are referred for this treatment. These patients are elderly, obese or both. As stated above, a common problem is their inability to undergo any form of anesthesia. Once it is decided that the patient is unfit for surgery, a work up it is necessary to establish the definitive radiation treatment. In order to do this, a pathology report is needed with information about the histological diagnosis and the differentiation grade (always present) of the tumor. CT-scan or MRI scan images are necessary to provide information about the tumor extension, specifically the depth of endometrial infiltration and extension of the tumor to adjacent organs, typically parametrium and vagina, and the lymph node status. Additionally, ultrasound of the vagina and endometrium provides information about the endometrial wall thickness and the depth of tumor infiltration.

The primary treatment choice is either brachytherapy alone or as a boost in combination with external beam. Generally, brachytherapy alone is considered for FIGO stage $1 \mathrm{~A}$ and $1 \mathrm{~B}$ grade 1 and 2 . Once there are indications for lymph node treatment: high grade histology and evidence of extensive myometrial involvement on MRI (FIGO 1B grade 3 and higher), a combination of external beam and brachytherapy has to be considered. The external beam therapy, if applicable, encompasses the entire uterus and cervix, at least the upper half of the vagina and lymph nodes up to the bifurcation of the common iliacs. The planned dose is 46-50.4 Gy in 23-28 fractions. The set-up of the brachytherapy procedure minimizes immobilization of the patient. The treatment consists of multiple applicator insertions, on an outpatient basis. If brachytherapy alone is given at ARTI, the patient receives 6 fractions of $6 \mathrm{~Gy}$. If brachytherapy is given as a boost, the patient receives 3 fractions of 7 Gy. The American

Table 3. HDR brachytherapy as primary treatment of endometrial cancer

\begin{tabular}{lcccc} 
Reference & Nr. patients & Applicator & Treatment & 5 year DSS \\
\hline Knocke et al. [26] & 235 & Single intrauterine & HDR \pm EBRT & $76 \%$ \\
\hline Weitmann et al. [27] & 16 & Heyman packing & HDR & $4-y$ r 100\% \\
\hline Coon et al. [24] & 49 & Y-shaped Rotte & HDR \pm EBRT & $87 \%$
\end{tabular}

Table 4. Suggested doses of HDR only for treatment of inoperable primary endometrial cancer [32]

\begin{tabular}{lccc} 
Nr. of HDR fractions & $\begin{array}{c}\text { HDR dose/fx* at } 2 \mathrm{~cm} \text { from midpoint of } \\
\text { intrauterine sources }\end{array}$ & $\begin{array}{c}\text { Equivalent dose for } \\
\text { tumor effects }\end{array}$ & $\begin{array}{c}\text { Equivalent dose } \\
\text { (late effects with DRF**) }\end{array}$ \\
\hline 4 & $8.5 \mathrm{~Gy}$ at $2 \mathrm{~cm}$ & 52.4 & 42.6 \\
\hline 5 & $7.3 \mathrm{~Gy}$ at $2 \mathrm{~cm}$ & 52.6 & 41.4 \\
\hline 6 & $6.4 \mathrm{~Gy}$ at $2 \mathrm{~cm}$ & 52.5 & 40.3 \\
\hline 7 & $5.7 \mathrm{~Gy}$ at $2 \mathrm{~cm}$ & 52.2 & 39.0
\end{tabular}

Table 5. Suggested doses of external beam + HDR for treatment of inoperable primary endometrial cancer [32]

\begin{tabular}{lcccc} 
EBRT (Gy) @ 1.8 Gy/fx & $\begin{array}{c}\text { Nr. of HDR } \\
\text { fractions }\end{array}$ & HDR dose/fx & $\begin{array}{c}\text { Equivalent dose for } \\
\text { tumor effects }\end{array}$ & $\begin{array}{c}\text { Equivalent dose } \\
\text { (late effects with DRF) }\end{array}$ \\
\hline 45 & 2 & 8.5 Gy at $2 \mathrm{~cm}$ & 70.5 & 64.5 \\
\hline 45 & 3 & 6.3 Gy at $2 \mathrm{~cm}$ & 69.9 & 62.8 \\
\hline 45 & 4 & 5.4 Gy at $2 \mathrm{~cm}$ & 70.6 & 62.5
\end{tabular}




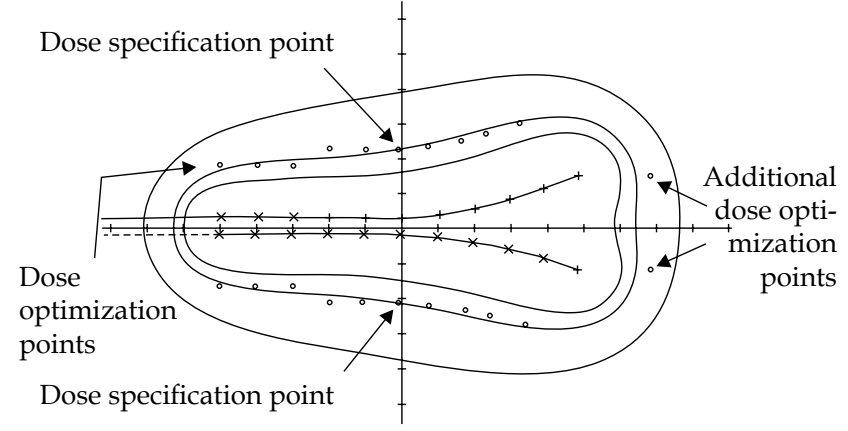

Fig. 1. A methodology for the planning procedure including specification and optimization points. The dose specification points are at a point $2 \mathrm{~cm}$ from the central axis at the midpoint along the uterine applicator. Additional optimization points are placed at $2 \mathrm{~cm}$ from the central axis of the tandem at its midpoint. The isodose is widened at the uterine fundus to account for the increased uterine width at the fundus
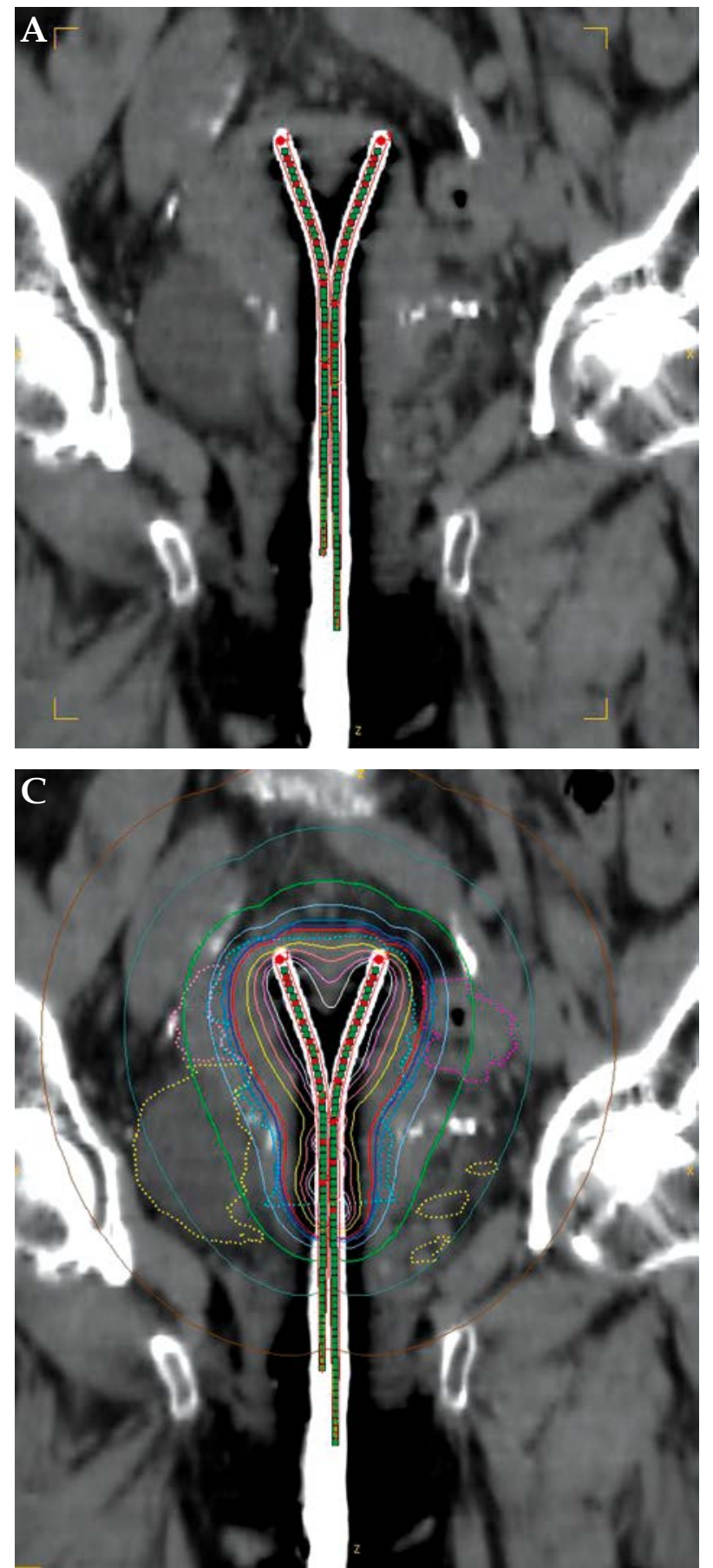

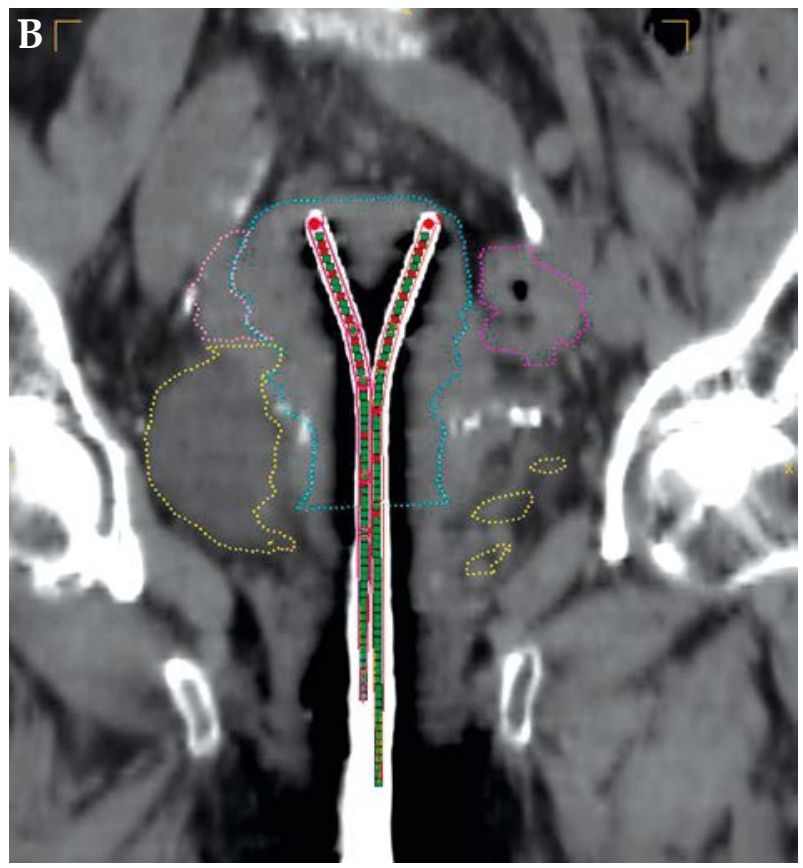

Fig. 2. A) CT-scan with Rotte applicator in the coronal view: The green and red points in the applicator are possible source positions. B) Contouring in the coronal view. The blue, pink, purple, and yellow contour lines are uterus, bowel, sigmoid, and urinary bladder, respectively. C) Definitive isodose distribution in the coronal view. The red isodose line illustrates $100 \%$ 

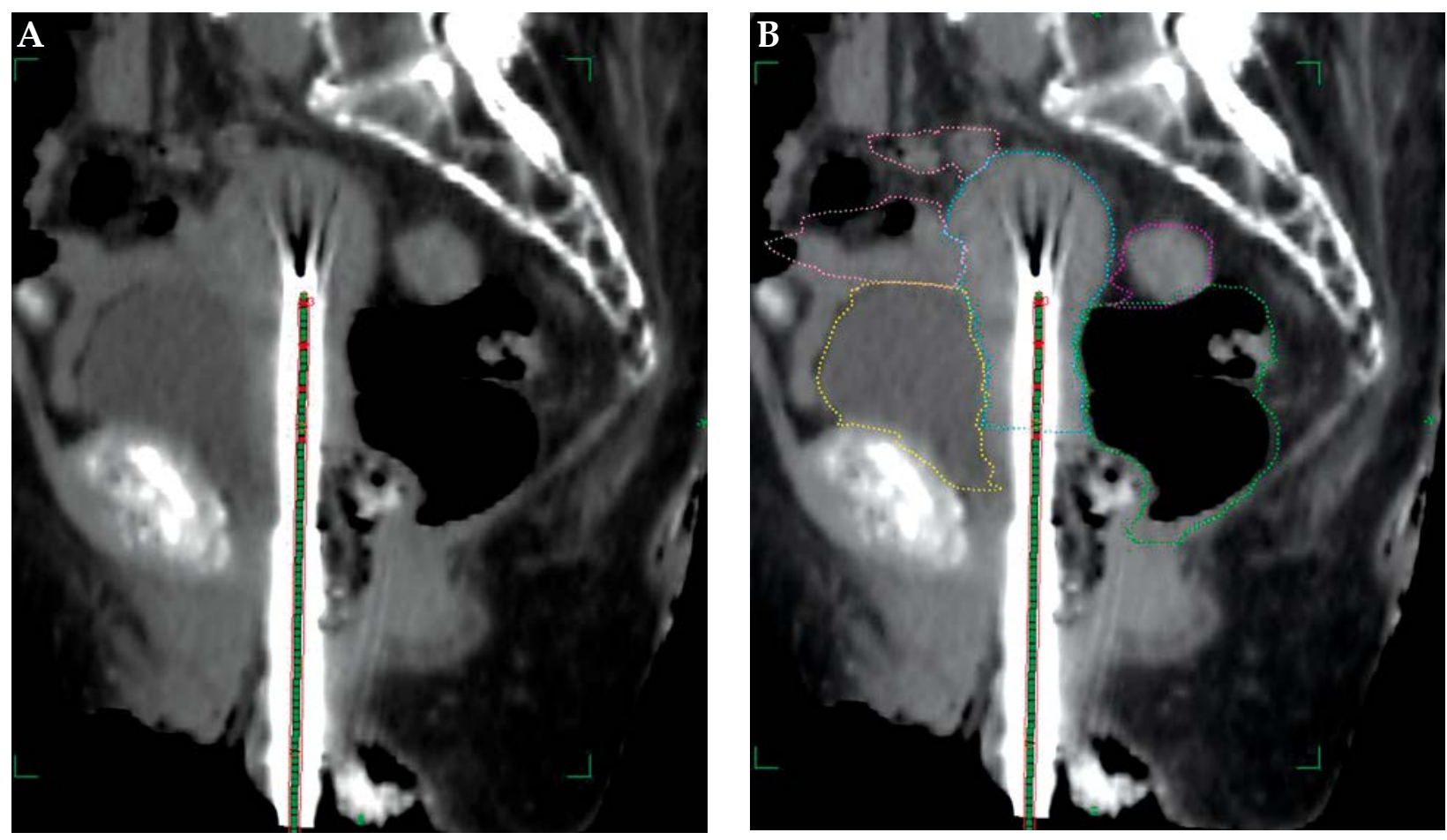

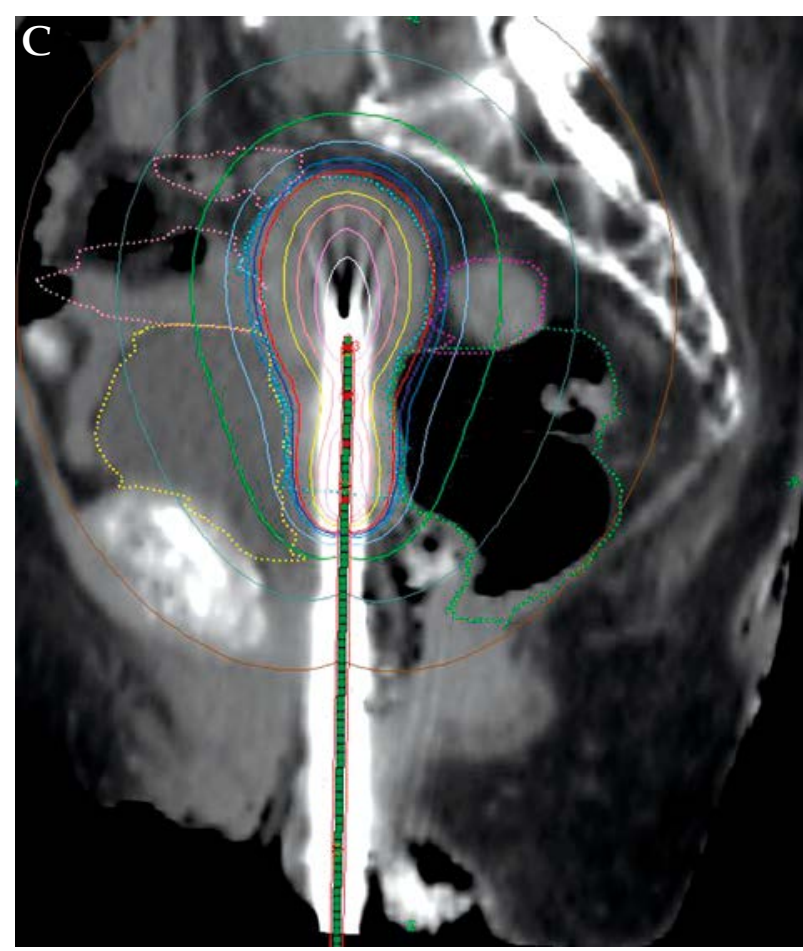

Brachytherapy Society has published treatment guidelines in 2000 [32] as cited below in Tables 4 and 5.

The entire brachytherapy procedure takes place in the treatment room. The patient is positioned on the gynecological bed in lithotomy position. The uterus is carefully probed using a soft plastic dilator. The applicator (in the appropriate size) is inserted, packed in position and fixed with a soft bandage. This out patient procedure can only be performed if the cervix and vagina are not involved. Post-implant imaging CT-scan is obtained immediately,
Fig. 3. A) CT scan with Rotte applicator in the sagittal view. The green and red points in the applicator are possible source positions. B) Contouring in the sagittal view. The blue, pink, purple, and yellow contour lines are uterus, bowel, sigmoid, and urinary bladder, respectively. C) Definitive isodose distribution in the sagittal view. The red isodose line illustrates $100 \%$

followed by contouring of the uterus (CTV), the tumor gross tumor volume (GTV), and organs at risk (OAR): bladder, rectum and sigmoid. Treatment plans are individualized for each patient. Nag et al. described a methodology for the planning procedure including specification and optimization points (Fig. 1) [32]. Image guided treatment planning secures further optimization.

We perform our planning accordingly. The ARTI planning procedure is best illustrated by a series of images (Figs. 2A-C, Figs. 3A-C and Figs. 4A-C), demonstrating 

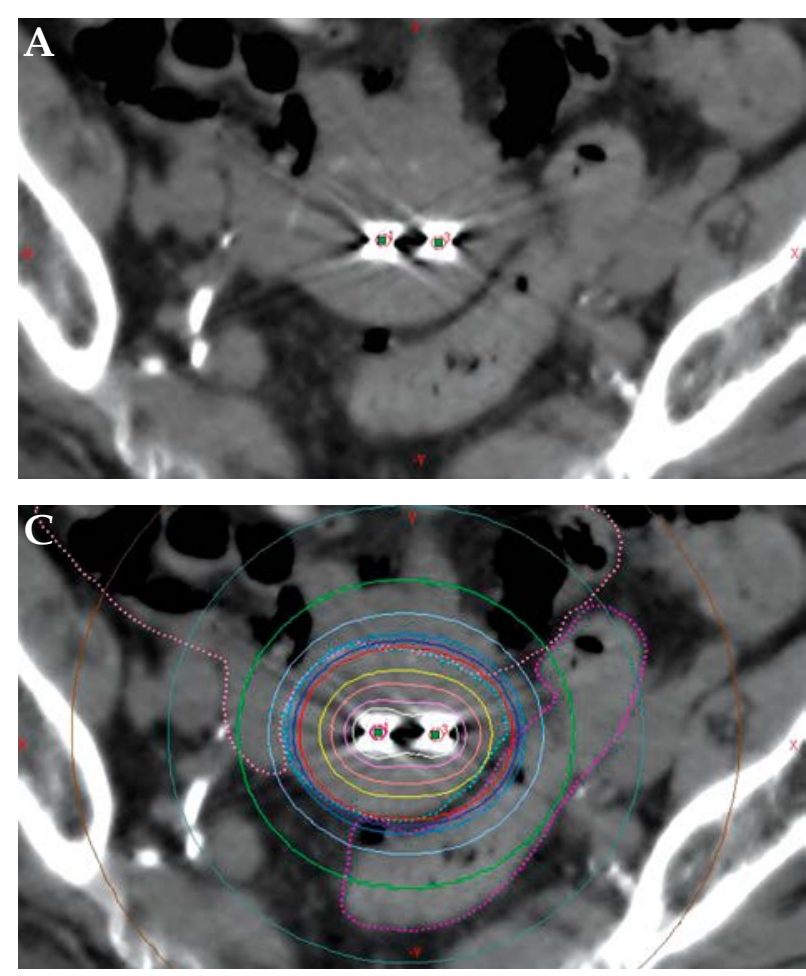

the consecutive steps: CT-scan, contouring and definitive isodose distribution in the coronal, sagittal, and transversal views.

By using 3D treatment planning we ensure to enclose the GTV with $100 \%$ isodose and intend to enclose the CTV with an isodose, which is as high as possible, keeping in mind that for OAR we intend not to exceed a cumulative isoeffective dose of D2cc for rectum of $75 \mathrm{~Gy}$ and for bladder of 80-85 Gy.

Each treatment is preceeded by a CT-scan and individual planning. After the planning the patient treatment follows. When finished, the applicator is removed and the patient can return home. Altogether, the patient spends two to three hours in our institution. The treatment is well tolerated by the patient. Fifty percent of patients developed acute toxicity in the form of enteritis CTCAE v4 grade 1 or 2 due to external beam radiation treatment, and one patient delevoped grade 2 small bowel late toxicity.

\section{Discussion}

Primary (image guided) brachytherapy based radiotherapy for endometrial cancer is an effective, well tolerated treatment approach. A multicenter study would help to develop and introduce a uniform protocol to achieve optimal results for this specific patient population. Future improvements of the applicator, in order to meet modern brachytherapy requirements, could include the development of an MRI compatible applicator, as image quality of the MRI is superior to CT. The GTV is almost never visible on $\mathrm{CT}$, the discrimination of pelvic structures is seen much better on MRI, especially distinction between the uterus and the bowel and the scatter of the applicator could be eliminated. If CT were to be replaced by MRI,

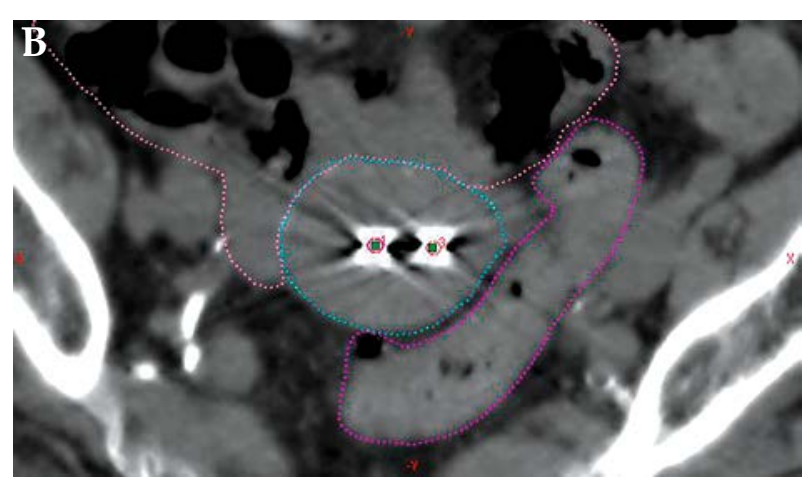

Fig. 4. A) CT scan with Rotte applicator in the transversal view. B) Contouring in the transversal view. The blue, pink, purple, and yellow contour lines are uterus, bowel, sigmoid and urinary bladder respectively. C) Definitive isodose distribution in the transversal view. The red isodose line illustrates $100 \%$

the delineations of all structures would be easier and the planning time could be significantly reduced, which would ultimately increase the patient comfort.

\section{Conclusions}

Primary (image guided) brachytherapy in inoperable endometrial cancer is an effective, well tolerated treatment approach with outcomes comparable to surgery. This approach might be considered more frequent in elderly and obese patients of any age for whom surgery may be associated with an increased risk of serious morbidity or even mortality.

\section{Disclosure}

Authors report no conflict of interest.

\section{References}

1. http://www.cancerresearchuk.org/cancer-help/type/womb; accessed March 2014.

2. Austin H, Austin JM Jr, Patridge EE et al. Endometrial cancer, obesity and body fat distribution. Cancer Res 1991; 51: 568-572.

3. http://www.cijfersoverkanker.nl/; accessed May 2014.

4. Benedetti PP, Basile S, Maneschi F et al. Systematic pelvic lymphadenectomy vs. no lymphadenectomy in early stage endometrial carcinoma: randomized clinical trial. J Natl Cancer Inst 2008; 100: 1707-1716.

5. Kitchener H, Swart AM, Qian Q et al. Efficacy of systematic pelvic lymphadenectomy in endometrial cancer (MRC ASTEC trial): a randomized study. Lancet 2009; 373: 125-136.

6. Creutzberg CL, van Putten WL, Koper PC et al. Surgery and postoperative radiotherapy versus surgery alone for patients with stage-1 endometrial carcinoma: multicenter randomized trial. PORTEC Study Group. Postoperative Radiation Therapy in Endometrial Carcinoma. Lancet 2000; 355: 1404-1411. 
7. Keys HM, Roberts JA, Brunetto VL et al. A phase III trial of surgery with or without adjunctive external pelvic radiation therapy in intermediate risk endometrial adenocarcinoma: a Gynecologic Oncology Group study. Gynecol Oncol 2004; 92: 744-751.

8. Aalders J, Abeler V, Kolstad P et al. Postoperative external irradiation and prognostic parameters in stage I endometrial carcinoma: clinical and histopathologic study of 540 patients. Obstet Gynecol 1980; 56: 419-427.

9. Blake P, Swart AM, Orton J et al. Adjuvant external beam radiotherapy in the treatment of endometrial cancer (MRC ASTEC and NCIC CTG EN.5 randomized trials): pooled trial results, systematic review, and meta-analysis. Lancet 2009; 373: 137-146.

10. Nout RA, Smit VT, Putter H et al. Vaginal brachytherapy versus pelvic external beam radiotherapy for patients with endometrial cancer of high-intermediate risk (PORTEC-2): an open label, non-inferiority, randomized trial. Lancet 2010; 375: 816-823.

11. Kellas-Ślęczka S, Wojcieszek P, Białas B. Adjuvant vaginal brachytherapy as a part of management in early endometrial cancer. J Contemp Brachytherapy 2012; 4: 247-252.

12. Donnelly E, Rakhra S, Helenowski I et al. Dosimetry and toxicity outcomes in postoperative high-dose-rate intracavitary brachytherapy for endometrial carcinoma J Contemp Brachytherapy 2012; 3: 135-140.

13. Hogberg T, Signorelli M, de Oliveira CF et al. Sequential adjuvant chemotherapy and radiotherapy in endometrial cancer-results from two randomized studies. Eur J Cancer 2010; 46: 2422-2431.

14. Creasman WT, Odicino F, Maisonneuve P et al. Carcinoma of the corpus uteri. FIGO 26th Annual Report on the Results of Treatment in Gynecological Cancer. Int J Gynaecol Obstet 2006; 95 Suppl 1: S105-143.

15. http://www.oncoline.nl/endometriumcarcinoom; accessed March 2014

16. Heyman J. The radiotherapeutic treatment of cancer corporis uteri. Br J Radiol 1947; 20: 85-91.

17. Jones DA, Stout R. Results of intracavitary radium treatment for adenocarcinoma of the body of the uterus. Clin Radiol 1986; 37: 169-171.

18. Grigsby PW, Kuske RR, Perez CA et al. Medically inoperable stage I adenocarcinoma of the endometrium treated with radiotherapy alone. Int J Radiat Oncol Biol Phys 1987; 13: $483-$ 488.

19. Taghian A, Pernot M, Hoffstetter S et al. Radiation therapy alone for medically inoperable patients with adenocarcinoma of the endometrium. Int J Radiat Oncol Biol Phys 1988; 15: 1135-1140.

20. Kupelian PA, Eifel PJ, Tornos C et al. Treatment of endometrial carcinoma with radiation therapy alone. Int J Radiat Oncol Biol Phys 1993; 27: 817-824.

21. Rose PG, Baker S, Kern M et al. Primary radiation therapy for endometrial carcinoma: a case controlled study. Int J Radiat Oncol Biol Phys 1993; 27: 585-590.

22. Rouanet $P$, Dubois J, Gely $S$ et al. Exclusive radiation therapy in endometrial carcinoma. Int J Radiat Oncol Biol Phys 1993; 26: 223-228.

23. Chao CK, Grisby PW, Perez CA et al. Brachytherapy-related complications for medically inoperable stage I endometrial carcinoma. Int J Radiat Oncol Biol Phys 1995; 31: 37-42.

24. Coon D, Beriwal S, Heron D et al. High-dose-rate Rotte "Y" applicator brachytherapy for definitive treatment of medically inoperable endometrial cancer: 10-year. Int J Radiat Oncol Biol Phys 2008; 71: 779-783.

25. Beriwal S, Kim H, Heron D et al. Comparison of 2D vs. 3D dosimetry for Rotte " $Y$ " applicator high dose rate brachyther- apy for medically inoperable endometrial cancer: 10-year. Technol Cancer Res Treat 2006; 5: 521-527.

26. Knocke TH, Kucer H, Weidinger B et al. Primary treatment of endometrial carcinoma with high-dose-rate brachytherapy: results of 12 years of experience with 280 patients. Int J Radiat Oncol Biol Phys 1997; 37: 359-365.

27. Weitmann HD, Potter R, Waldhause C et al. Pilot study in the treatment of endometrial carcinoma with $3 \mathrm{D}$ image-based high-dose-rate brachytherapy using modified Heyman packing: clinical experience and dose-volume histogram analysis. Int J Radiat Oncol Biol Phys 2005; 62: 468-478.

28. Gerszten K, Faul C, Kelley J et al. Twice-daily high-rate brachytherapy for medically inoperable uterine cancer. Brachytherapy 2006; 5: 118-121.

29. Inciura A, Aktocius V, Juozaityte E et al. Long-term results of high-dose-rate brachytherapy and external-beam radiotherapy in the primary treatment of endometrial cancer. J Radiat Res 2010; 51: 675-681.

30. Niazi TM, Souhami L, Portelance L et al. Long-term results of high-dose-rate brachytherapy in the primary treatment of medically inoperable stage I-II endometrial carcinoma. Int J Radiat Oncol Biol Phys 2005; 63: 1108-1113.

31. Gerbaulet A, Pötter R, Mazeron JJ et al. The GEC ESTRO handbook of brachytherapy. ACCO, Leuven 2002.

32. Nag S, Erickson B, Parikh S et al. The American Brachytherapy Society recommendations for high-dose-rate brachytherapy for carcinoma of the endometrium. Int J Radiat Oncol Biol Phys 2000; 48: 779-790. 